\title{
Experimental Study on the Radiative Properties of Fly Ash in the Radiant Syngas Cooler of Gasifier
}

\author{
Zhifei Fu, Qian Wang, Jiansheng Zhang \\ Department of Energy and Power Engineering, Tsinghua University, Beijing, China \\ Email: fzf16@mails.tsinghua.edu.cn
}

How to cite this paper: Fu, Z.F., Wang, Q. and Zhang, J.S. (2018) Experimental Study on the Radiative Properties of Fly Ash in the Radiant Syngas Cooler of Gasifier. Journal of Power and Energy Engineering, 6, 9-20.

https://doi.org/10.4236/jpee.2018.69002

Received: July 24, 2018

Accepted: September 4, 2018

Published: September 7, 2018

\begin{abstract}
Radiant syngas cooler (RSC) is the key heat recovery equipment in coal gasification system. The syngas from gasifier carries large amount of slags in which the mass fraction of fly ash less than $100 \mu \mathrm{m}$ is about $20 \%$. Studying the optical properties of fly ash has high significance for the optimization of heat transfer calculation in RSC. A new experimental method was proposed to inversely calculate the radiative parameters of particles-" $\mathrm{KBr}$ transmittance-reflectance method". By measuring the "directional-hemispherical" reflectance and transmittance of fly ash particles by FTIR under the wavelength range of 0.55 - $1.65 \mu \mathrm{m}$, using the four-flux model to solve the radiative transfer equation and combing with Mie theory, the absorption and scattering efficiency of 22.7 $\mu \mathrm{m}$ fly ash and optical constant (also known as complex refractive index, $m=$ $n+i k)$ of fly ash were inversely calculated. The results indicated that for fly ash with large size parameter, there was no obvious change of the absorption and scattering efficiency when the mass fraction of $\mathrm{Fe}_{2} \mathrm{O}_{3}$ was between $5.65 \%$ and $16.53 \%$, which was well explained by Mie theory; The obtained optical constant was close to the results of $\mathrm{KBr}$ transmittance method.
\end{abstract}

\section{Keywords}

Radiant Syngas Cooler, Fly Ash, Radiative Properties, Absorption Efficiency, Scattering Efficiency, Optical Constant

\section{Introduction}

The syngas produced by various gasifiers contains about $10 \%$ to $20 \%$ calorific value which exists in the form of sensible heat. Usually, this part of the energy is not effectively used. For example, the high-temperature syngas in coal-water 
slurry gasifier is directly cooled by chilling. The syngas temperature decreases from about $1400^{\circ} \mathrm{C}$ to less than $300^{\circ} \mathrm{C}$, which causes huge energy loss. Radiant Syngas Cooler (RSC) is installed at the outlet of gasifier and plays a significant role in recovering this part of energy. The utilization of RSC can increase the power generation efficiency of Integrated Gasification Combined Cycle (IGCC) by 4 - 5 percents [1].

The syngas flowing into RSC carries a large amount of high-temperature slags, in which the mass fraction of particles less than $100 \mu \mathrm{m}$ is about $20 \%$. Because of its small size and large number density, this part of fly ash has a significant effect of light scattering on the radiation transfer in RSC [2]. Therefore, the study of the optical properties of fly ash, including its absorption efficiency, scattering efficiency and optical constant, is important to the optimization of heat transfer in RSC.

The optical properties of fly ash are determined by chemical composition, size parameter $x(x=\pi D / \lambda)$, surface condition and geometry [3]. In view of the radiative properties of fly ash, a lot of researches have been carried out mainly through the combination of calculation and experiment. Goodwin examined the optical constant of slags in the wavelength of $0.19-13 \mu \mathrm{m}$ by measuring the the near normal reflectance and transmittance, which is widely accepted [4] [5]. Self and Ruan determined the optical constant by measuring $\mathrm{KBr}$ slabs that contain fly ash particles and then combining with Mie theory and K-K relation, but the extrapolation formula of the K-K relation will cause some deviations [6] [7] [8]. Meanwhile, the result of extinction coefficient $k$ through this method is $10-100$ times larger than that given by Goodwin. This is because particles have larger surface areas than bulk [9]. Yin and Liu obtained effective optical constant of fly ash based on the effective medium theory (EMT) of mean electromagnetic field [10]. Self compared the optical constant of slag from $300 \mathrm{~K}$ to $1900 \mathrm{~K}$. It was found that the $\mathrm{k}$ value of some wavelength increased by nearly three times [6]. Mengüc and Wall used light scattering method to measure the optical constant of coal and fly ash respectively, but this method was difficult to apply [11] [12].

In order to eliminate the errors from the extrapolation formula of the K-K relation and reduce the effects of forward scattering in $\mathrm{KBr}$ transmittance method, a "KBr transmittance-reflectance method" was proposed in this paper based on [13] [14] [15] to obtain the optical constant of $3.21 \mu \mathrm{m}$ fly ash particles and the absorption and scattering efficiency of $24.8 \mu \mathrm{m}$ fly ash particles.

\section{Theoretical Model}

\subsection{Lorenz-Mie Theory}

Lorenz-Mie theory is a classical algorithm used to solve the interaction between spherical particles and plane electromagnetic waves. It gives an exact solution to the scattering problem of spherical particles with any size. When a light with wavelength of $\lambda$ is incident on an isotropic homogeneous spherical particle, the extinction, scattering and absorption efficiency of the particle can be expressed by the following formula [16] [17]: 


$$
\begin{gathered}
Q_{\text {ext }}(m, x)=\frac{2}{x^{2}} \sum_{n=1}^{\infty}(2 n+1) \operatorname{Re}\left\{a_{n}+b_{n}\right\} \\
Q_{s c a}(m, x)=\frac{2}{x^{2}} \sum_{n=1}^{\infty}(2 n+1)\left[\left|a_{n}\right|^{2}+\left|b_{n}\right|^{2}\right] \\
Q_{a b s}(m, x)=Q_{\text {ext }}(m, x)-Q_{s c a}(m, x)
\end{gathered}
$$

where $x$ is size parameter; $r$ is particle radius; $a_{n}$ and $b_{n}$ are called Mie scattering coefficients, which are calculated as follows:

$$
\begin{aligned}
& a_{n}=\frac{\psi_{n}^{\prime}(m x) \psi_{n}(x)-m \psi_{n}(m x) \psi_{n}^{\prime}(x)}{\psi_{n}^{\prime}(m x) \xi_{n}(x)-m \psi_{n}(m x) \xi_{n}^{\prime}(x)} \\
& b_{n}=\frac{m \psi_{n}^{\prime}(m x) \psi_{n}(x)-\psi_{n}(m x) \psi_{n}^{\prime}(x)}{m \psi_{n}^{\prime}(m x) \xi_{n}(x)-\psi_{n}(m x) \xi_{n}^{\prime}(x)}
\end{aligned}
$$

where $m=n+i k$ represents the optical constant; $\xi_{n}=\psi_{n}-i \eta_{n} ; \psi_{n}$ and $\eta_{n}$ are the Bessel functions which follow the recurrence relation:

$$
\begin{aligned}
& \psi_{n+1}(z)=\frac{2 n+1}{z} \psi_{n}(z)-\psi_{n-1}(z) \\
& \eta_{n+1}(z)=\frac{2 n+1}{z} \eta_{n}(z)-\eta_{n-1}(z)
\end{aligned}
$$

where,

$$
\begin{aligned}
& \psi_{-1}(z)=\cos z, \quad \psi_{0}(z)=\sin z \\
& \eta_{-1}(z)=-\sin z, \quad \eta_{0}(z)=\cos z
\end{aligned}
$$

For dispersed particle system that satisfies the independent scattering condition, the scattering of particles has no effect on each other. The absorption and scattering coefficients of the particle system are the integral of the absorption and scattering efficiency by the particle size distribution, as follows:

$$
\mu_{\lambda}=\int_{0}^{\infty} Q_{\mu} \frac{\pi D^{2}}{4} f(D) N d D
$$

where $\mu_{\lambda}$ represents the absorption coefficient scattering $\kappa_{\lambda}$, scattering coefficient $\sigma_{\lambda}$, extinction coefficient $\sigma_{\lambda} ; Q_{\mu}$ represents the corresponding absorption, scattering and extinction efficiency; $N$ denotes the number of particles per unit volume; $f(D)$ is the normalized size distribution. For particles with same size, the relation is simplified as:

$$
\mu_{\lambda}=\frac{\pi D^{2}}{4} N Q_{\mu}
$$

Therefore, when the particle absorption, scattering coefficients and the size distribution are obtained from experiments, the absorption, scattering efficiency and optical constants of particles can be inversely calculated.

\subsection{Four-Flux Model}

The four-flux model is an approximate solution of radiative transfer equation to 
determine the absorption and scattering coefficients of materials. In four-flux model, it is assumed that 1) there is no emission of the sample at room temperature; 2) only a collimated radiative flux is incident onto the front surface of the sample; 3) at any position $\mathrm{z}$ inside of the sample, there are four parts of energy: a collimated beam $I_{c}(z)$ propagating to positive $\mathrm{z}$, a collimated beam $J_{c}(z)$ propagating to negative $\mathrm{z}$, a diffuse beam $I_{d}(z)$ propagating to positive $\mathrm{z}$, a diffuse beam $J_{d}(z)$ propagating to negative $\mathrm{z}[18][19]$.

As shown in Figure 1, 0 L denotes the $\mathrm{KBr}$ slab that contains fly ash particles; $\rho_{\text {in }}$ and $\rho_{\text {out }}$ represent the internal and external reflectivity respectively which can be calculated by Fresnel equation [19]: $\rho_{\text {in }}=0.6299, \rho_{\text {out }}=0.1004$.

According to energy balance, the differential equations and boundary conditions can be established, see [20]. In this paper, the total reflectance $R$ and transmittance $T$ at two planes of 0 and $\mathrm{L}$ are derived under diffuse reflection boundary condition. The formulas are as follows:

$$
\begin{gathered}
R=\rho_{\text {out }}+\left(1-\rho_{\text {in }}\right)\left[C_{2}+C_{1} B+C_{2} A+C_{3}(1-\beta)+C_{4}(1+\beta)\right] \\
T=\left(1-\rho_{\text {in }}\right)\left[C_{1} e^{-\tau_{1}}+C_{1} A e^{-\tau_{1}}+C_{2} B e^{\tau_{1}}+C_{3}(1+\beta) e^{-2 \beta \tau_{2}}+C_{4}(1-\beta) e^{2 \beta \tau_{2}}\right]
\end{gathered}
$$

The constants in the formulas are found to be:

$$
\begin{gathered}
C_{1}=\frac{1-\rho_{\text {out }}}{1-\rho_{\text {in }}^{2} e^{-2 \tau_{1}}} \\
C_{2}=\frac{\left(1-\rho_{\text {out }}\right) \rho_{\text {in }} e^{-2 \tau_{1}}}{1-\rho_{\text {in }}^{2} e^{-2 \tau_{1}}}
\end{gathered}
$$

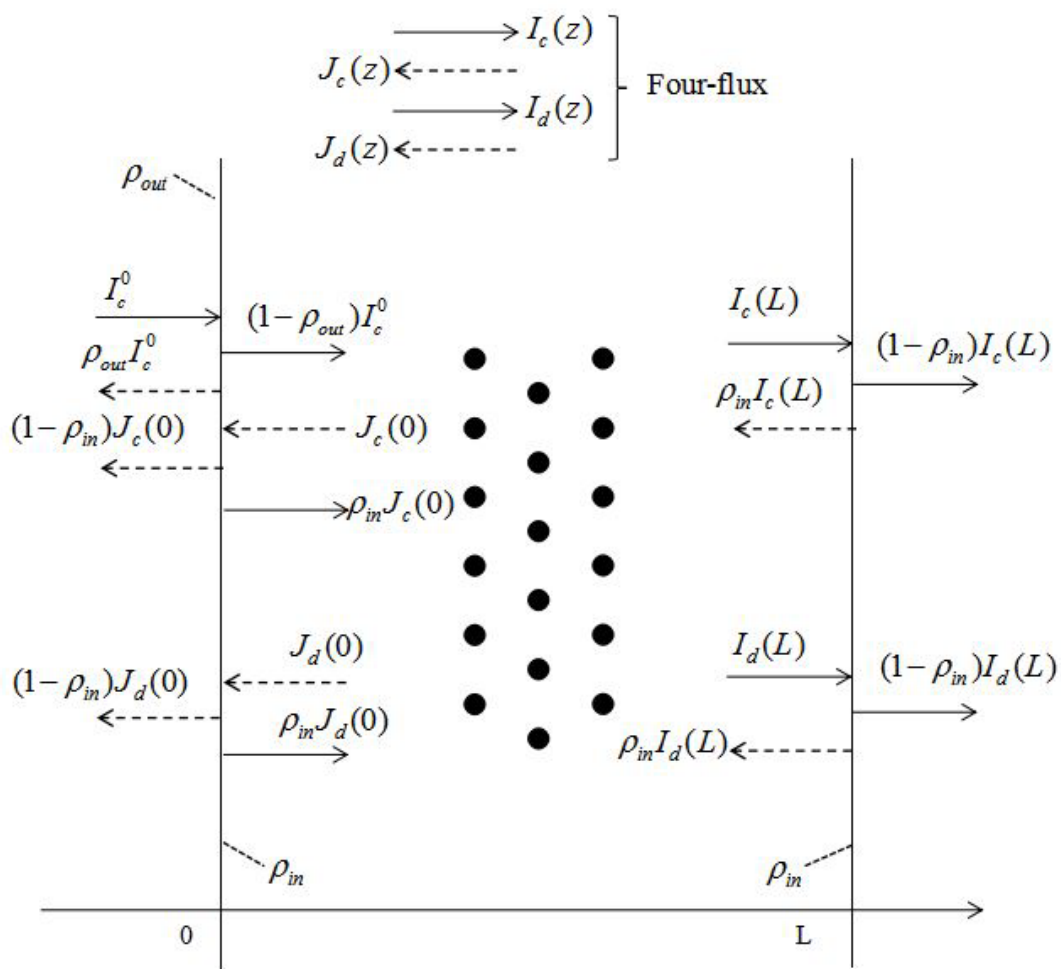

Figure 1. Illustration of four-flux model. 


$$
\begin{aligned}
C_{3} & =\frac{\left(\sigma_{1} \mu_{2} e^{2 \beta \tau_{2}}-\sigma_{2} \mu_{1} e^{-\tau_{1}}\right) C_{1}+\left(\sigma_{1} \mu_{1} e^{2 \beta \tau_{2}}-\sigma_{2} \mu_{2} e^{\tau_{1}}\right) C_{2}}{\sigma_{1}^{2} e^{2 \beta \tau_{2}}-\sigma_{2}^{2} e^{-2 \beta \tau_{2}}} \\
C_{4} & =\frac{\left(\sigma_{1} \mu_{1} e^{-\tau_{1}}-\sigma_{2} \mu_{2} e^{-2 \beta \tau_{2}}\right) C_{1}+\left(\sigma_{1} \mu_{2} e^{\tau_{1}}-\sigma_{2} \mu_{1} e^{-2 \beta \tau_{2}}\right) C_{2}}{\sigma_{1}^{2} e^{2 \beta \tau_{2}}-\sigma_{2}^{2} e^{-2 \beta \tau_{2}}}
\end{aligned}
$$

where,

$$
\begin{gathered}
\tau_{1}=(a+s) L ; \tau_{2}=0.5(M+N) L ; \quad \mu_{1}=\rho_{i n} A-B ; \quad \mu_{2}=\rho_{i n} B-A ; \\
M=\varepsilon_{d}[a+(1-\zeta) s] ; \quad N=\varepsilon_{d}(1-\zeta) s ; X=\varepsilon_{c}(1-\zeta) s ; Y=\varepsilon_{c} \zeta s ; T=a+s ; \\
\sigma_{1}=(1+\beta)-\rho_{i n}(1-\beta) ; \sigma_{2}=(1-\beta)-\rho_{i n}(1+\beta) \\
A=\frac{Y(M+T)+N X}{M^{2}-T^{2}-N^{2}} ; B=\frac{X(M-T)+N Y}{M^{2}-T^{2}-N^{2}} ; \beta=\frac{\sqrt{M^{2}-N^{2}}}{M+N} .
\end{gathered}
$$

where, $a$ and $s$ are the absorption and scattering coefficient respectively, and because $\mathrm{KBr}$ has no absorption in the infrared and partial visible spectrum, $a$ and $s$ are approximately treated as the absorption and scattering coefficient of the fly ash particles; $L$ is the thickness of sample; $\zeta$ is the forward scattering ratio, which equals the energy scattered by a particle in the forward hemisphere over the total energy; $\varepsilon$ is the average crossing parameter, which is defined by saying that, when the radiation light travels a length $d z$, the average path length is $\varepsilon d z$ [20]. For collimated light, $\varepsilon_{c}=1$ and diffuse light is indicated by $\varepsilon_{d}$.

In order to make sure the accuracy of the four-flux model, the selection of $\varepsilon_{d}$ and $\zeta$ is particularly important. Since the size distribution of particles can be controlled in experiment, $\varepsilon_{d}$ and $\zeta$ are calculated under different size parameter $x$ and $k$ by Mie scattering theory, see Figure 2.

As shown in Figure $2, \varepsilon_{d}$ and $\zeta$ change little under different $k$ values at the size parameter $x<1$. However, at the size parameter $1<x<10$, there is significant enhancement of forwarding scattering of particles, thus $\varepsilon_{d}$ and $\zeta$ have sharp changes with different $k$ values. As size parameter $x$ increases larger than $10, \varepsilon_{d}$ and $\zeta$ reaches constants respectively, which are $1.05 \%$ and $95 \%$. Because the particle sizes in experiments are $22.7 \mu \mathrm{m}$ and $3.21 \mu \mathrm{m}$ and the measuring wavelength is $0.55-1.65 \mu \mathrm{m}$, the size parameters are larger than 6 . Thus in calculation, $\varepsilon_{d}$ and $\zeta$ are chosen to be $\varepsilon_{d}=1.1$ and $\zeta=0.95$.

\section{Experimental Procedures}

\subsection{Sample Preparation}

The preparation of samples plays a crucial role in the experimental results. In the experiment, fully ground $\mathrm{KBr}$ was mixed with fly ash particles and then compressed into slab. The procedures of sample preparation are as follows: 1) Spectrum pure $\mathrm{KBr}$ pellets are ground into powder; 2) Fly ash and $\mathrm{KBr}$ are mixed with mass ratio $1: 100 ; 3)$ The mixture was compressed using a tablet pressing machine under pressure $7 \mathrm{t} / \mathrm{cm}^{2}$; 4) Because $\mathrm{KBr}$ is deliquescent, infrared heating was used to ensure that the preparation of sample was in a dry environment. 

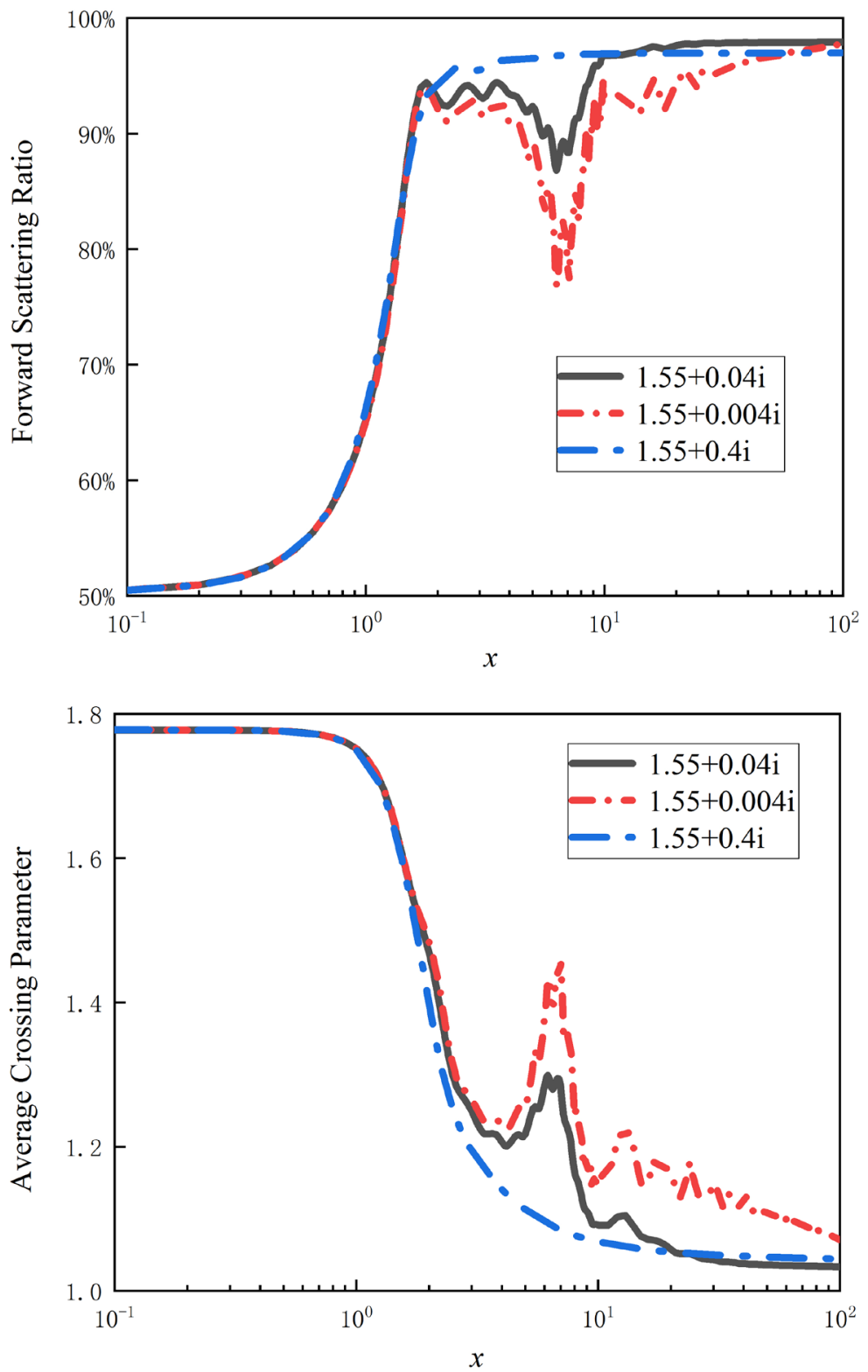

Figure 2. Forward scattering ratio and average crossing parameter calculated by Mie theory.

\subsection{Experimental Method}

Measurement of the hemispherical-directional reflectance and transmittance spectra of free-standing $\mathrm{KBr}$ sample was performed at room temperature, which means the emission of the sample can be neglected. The experiments examined the wavelength range of $0.55-1.65 \mu \mathrm{m}$ using Fourier Transform Infrared Spectroscopy (FTIR). The experiment equipment illuminated hemispherical radiation onto sample slab. Then the effective radiation intensity of upper surface $I_{e f f, 1}$ and lower surface $I_{e f f, 2}$ were separately received by two FTIRs, see Figure 3. Since the radiation intensity from the hemispherical radiation source $I_{\lambda, \text { source }}$ was known, the reflectance $R$ and transmittance $T$ were readily calculated as 


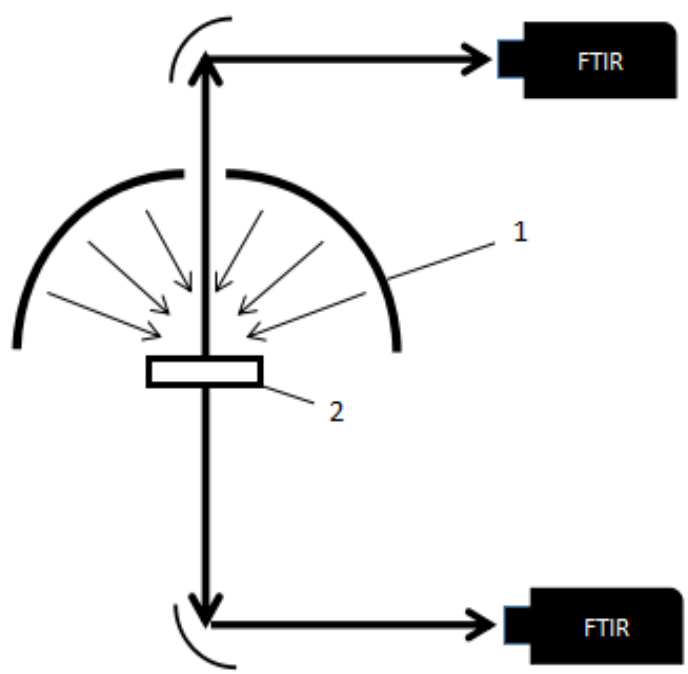

Figure 3. Illustration of experiment equipment ( 1 is a hemispherical radiation source, 2 is $\mathrm{KBr}$ slab).

$R=I_{\text {eff }, 1} / I_{\lambda, \text { source }}, T=I_{e f f, 2} / I_{\lambda, \text { source }}$. According to the reversibility of light, the hemispherical-directional reflectance $R$ and transmittance $T$ were equal to directional-hemispherical reflectance and transmittance that are required in the four-flux model. Based on the experiment data, the absorption, scattering coefficients of the fly ash system can be calculated and then the absorption, scattering efficiency and optical constant of single particle can be further retrieved.

\section{Results and Discussion}

\subsection{Optical Constant of $3.21 \mu \mathrm{m}$ Fly Ash Particle}

There is multiple value problem when calculating optical constant of particles with large size parameter, which means a group of testing data $(R, T)$ will get multiple values of $(n, k)$. Thus, the optical constant of fly ash was retrieved from particles $\bar{D}_{43}=3.21 \mu \mathrm{m}$. The reflectance and transmittance spectra are displayed in Figure 4. The calculated optical constant is shown in Figure 5.

As shown in Figure 5, $n$ value is essentially in agreement with the reference data which is between 1.5 and 1.6. The $k$ value is similar to the results of $\mathrm{KBr}$ transmission method in reference [21], and is quite different from the result of slag in reference [4]. This is because particles have larger surface area than bulk materials, so that the measured $k$ value of fly ash is larger than that of slag.

In the calculation of the heat transfer in RSC, the absorption, scattering coefficient and phase function of the fly ash particle system can be obtained according to the optical constant combining with suitable particle scattering model and particle size distribution, and the radiative transfer equation can be further solved.

\subsection{Absorption, Scattering Efficiency of $22.7 \mu \mathrm{m}$ Fly Ash Particle}

In the experiment, the density of fly ash was $\rho=2225 \mathrm{~kg} / \mathrm{m}^{3}$; the thickness of 


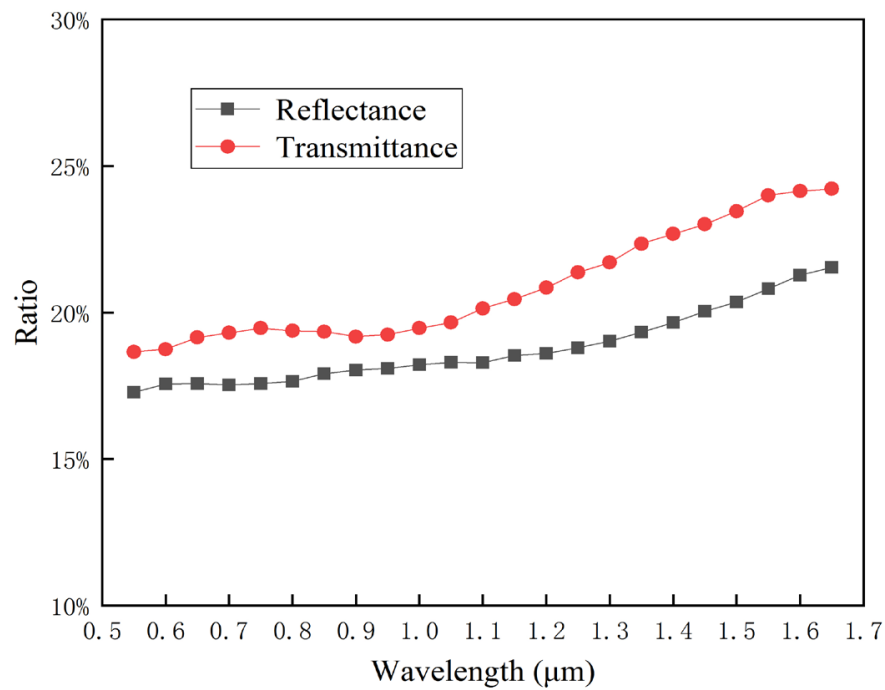

Figure 4. Reflectance and transmittance spectra of $3.21 \mu \mathrm{m}$ fly ash.
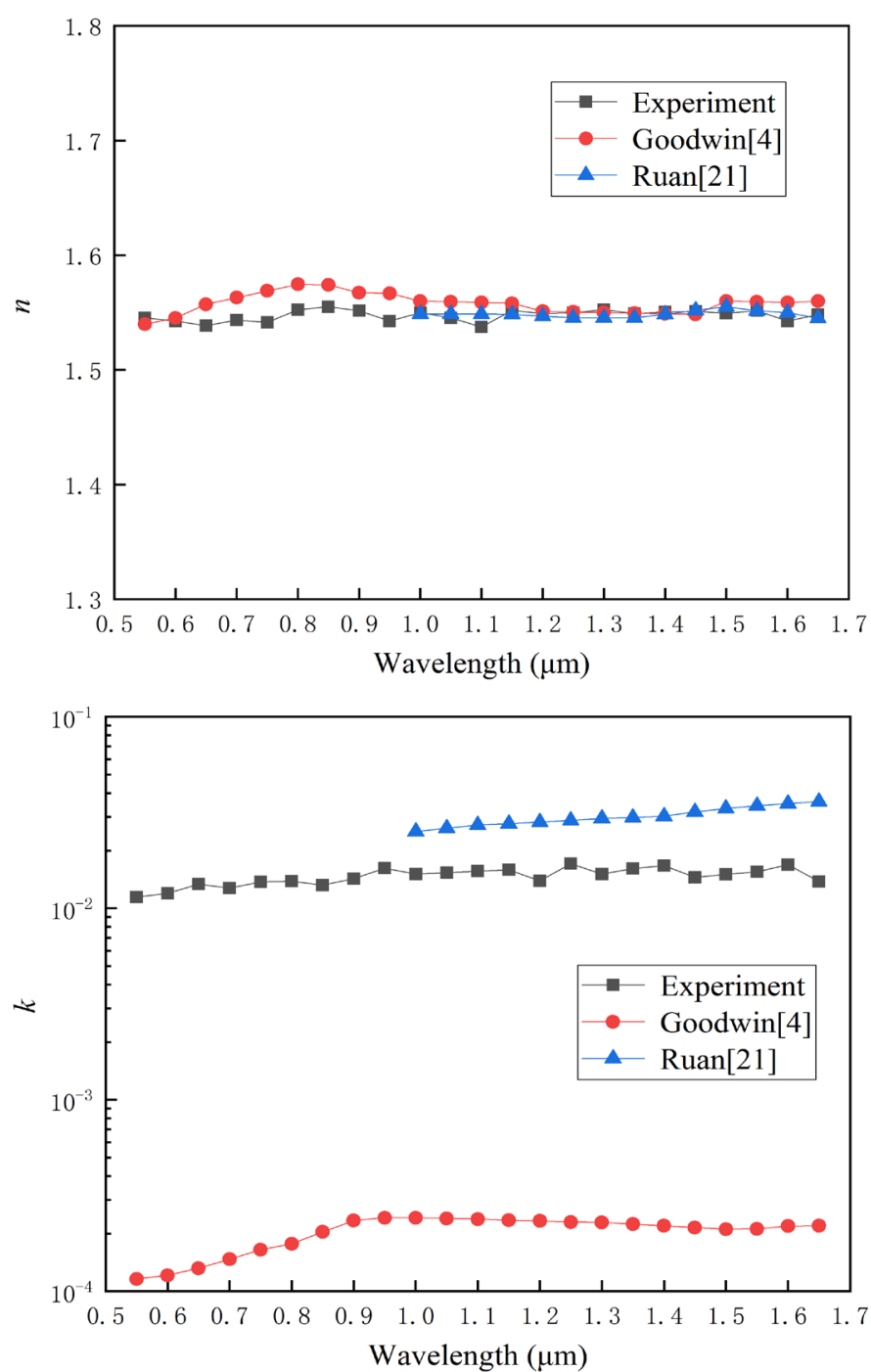

Figure 5. Optical constant of $3.21 \mu \mathrm{m}$ fly ash. 

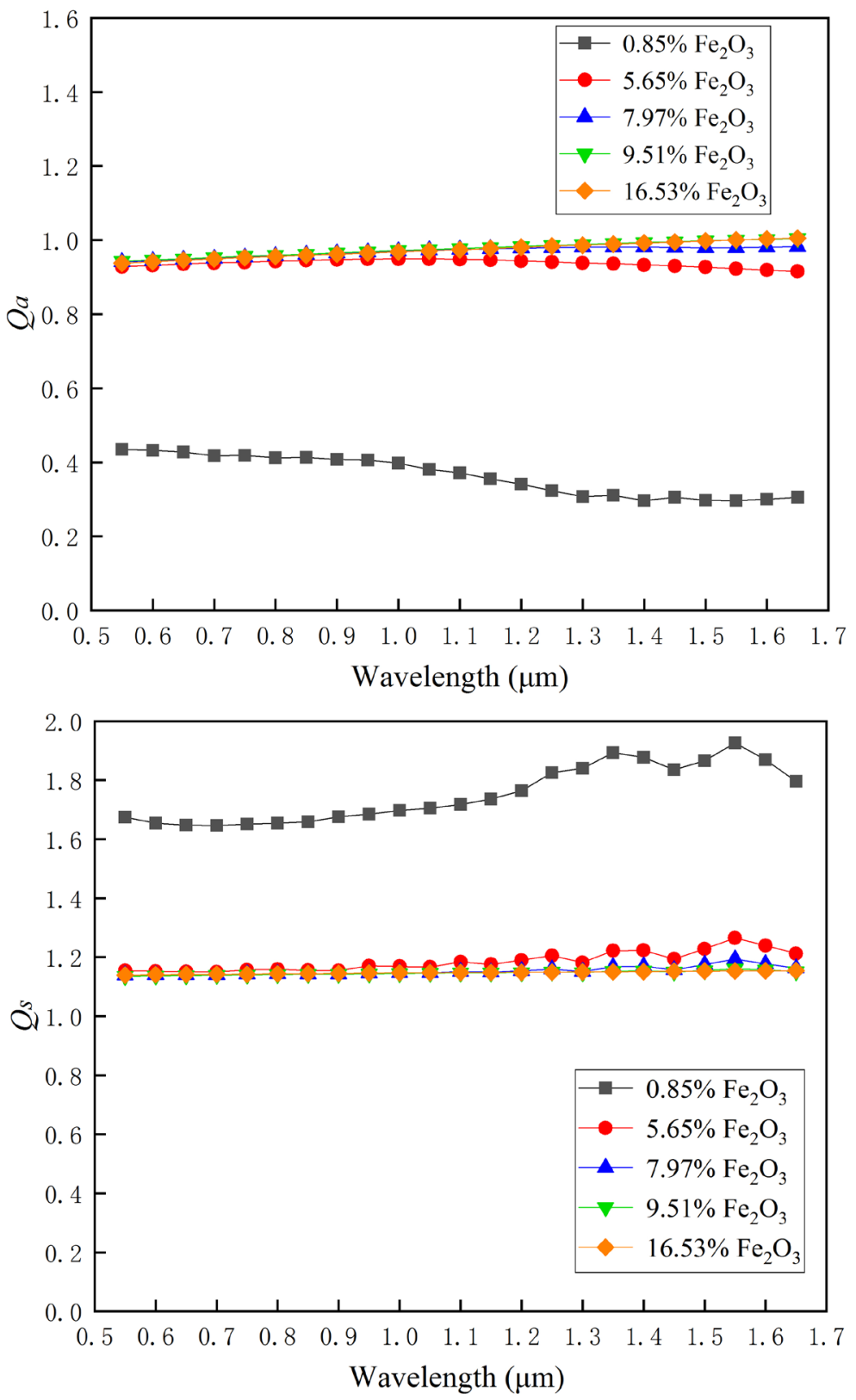

Figure 6. Absorption efficiency $Q_{a}$ and scattering efficiency $Q_{s}$ of $22.7 \mu \mathrm{m}$ fly ash.

KBr sample was $L=1.1 \mathrm{~mm}$; particle size was $\bar{D}_{43}=22.7 \mu \mathrm{m}$. Five groups of controlled experiment were set up with the mass fraction of $\mathrm{Fe}_{2} \mathrm{O}_{3}$ ranging from $0.85 \%$ to $16.53 \%$. The absorption and scattering efficiency calculated by genetic algorithm is shown in Figure 6.

It can be seen from Figure 6 that when the mass fraction of $\mathrm{Fe}_{2} \mathrm{O}_{3}$ was between $5.65 \%$ and $16.53 \%$, the change of $Q_{a}$ and $Q_{s}$ was not obvious and they concentrated around 0.9 and 1.1 respectively. Only when the mass fraction of $\mathrm{Fe}_{2} \mathrm{O}_{3}$ was reduced to $0.85 \%, Q_{a}$ and $Q_{s}$ changed significantly: $Q_{a}$ decreased to 0.3 - 0.4, while $Q_{s}$ increased to 1.7 - 1.8 .

Since $\mathrm{Fe}_{2} \mathrm{O}_{3}$ has strong absorption characteristic when the incident wavelength 
is less than $4 \mu \mathrm{m}$ [4], $k$ is mainly influenced by the content of $\mathrm{Fe}_{2} \mathrm{O}_{3}$ and increases with the increase of $\mathrm{Fe}_{2} \mathrm{O}_{3}$, while the $n$ value remains stable. Thus it is worth analyzing the results in Figure 6 by comparing the change of $Q_{a}$ and $Q_{s}$ under different size parameter $x$ and $k$ values through Mie scattering theory, see Figure 7.

As shown in Figure 7, with the increase of size parameter $x$, the influence of $k$ value on particle scattering and absorption efficiency gradually decreases; when $k$ grows larger, the values of $Q_{a}$ and $Q_{s}$ coincide. In the experiment, when the mass fraction of $\mathrm{Fe}_{2} \mathrm{O}_{3}$ was relatively high, the $k$ value became larger, so the change of $Q_{a}$ and $Q_{s}$ was not obvious as shown in Figure 6. In addition, it is
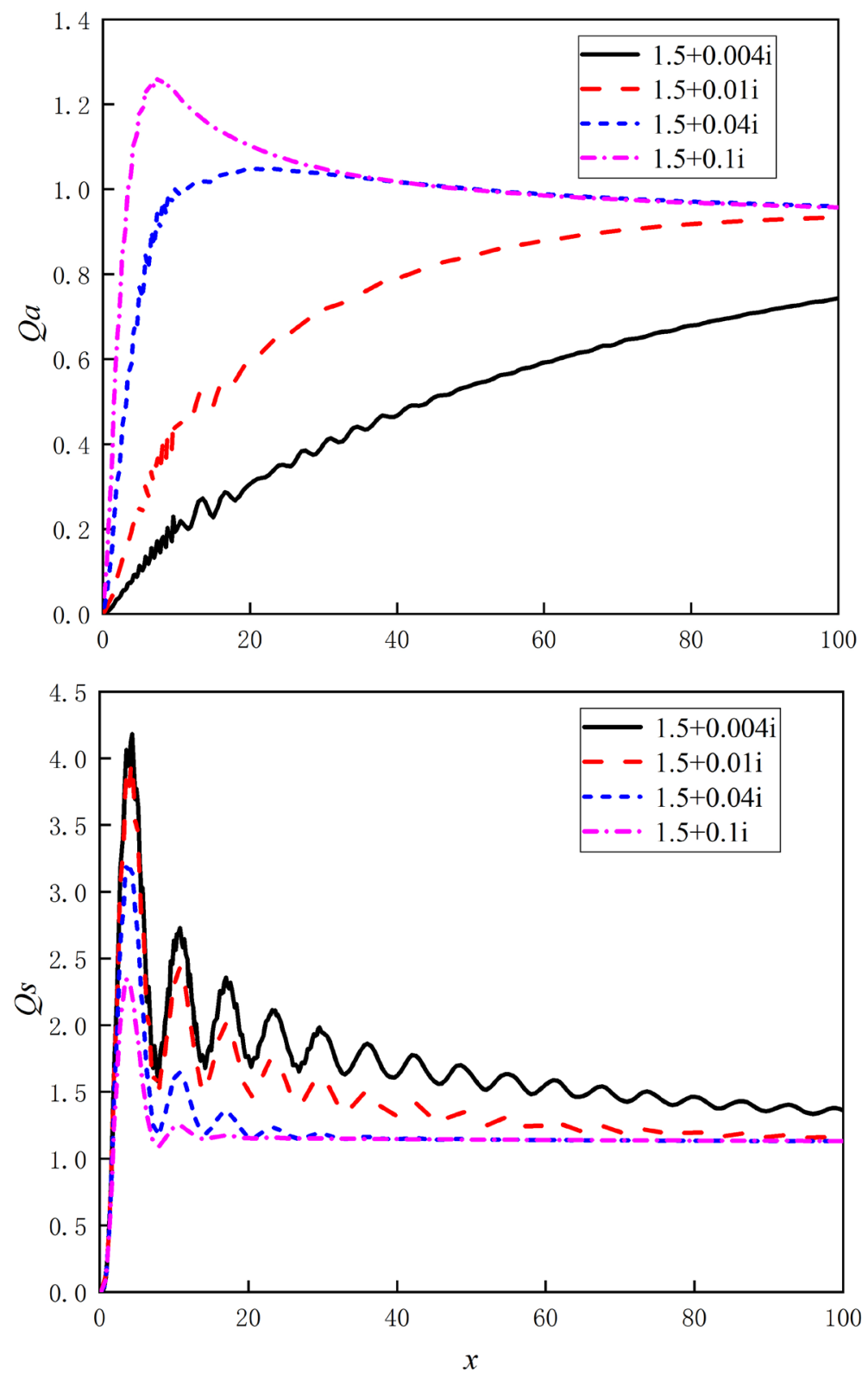

Figure 7. Direct calculation of absorption efficiency $Q_{a}$ and scattering efficiency $Q_{s}$ by Mie theory. 
found that with the increase of the size parameter $x$, the scattering and absorption efficiency of the particles approach a constant respectively. And for materials with larger $k$ value, the size parameter $x$ is smaller when the absorption and scattering efficiency tends to be stable.

In the calculation of heat transfer in RSC, the absorption and scattering efficiency of large size parameter fly ash can be approximately determined as fixed values which are not affected by the variation of $\mathrm{Fe}_{2} \mathrm{O}_{3}$.

\section{References}

[1] Ni, J.J. (2010) Investigation of Multiphase Flow, Heat Transfer and the Behavior of Molten Slag in Entrained-Flow Gasifier and Its Radiant Syngas Cooler. Ph.D. Thesis, East China University of Science and Technology, Shanghai.

[2] Li, X.B., Guo, Q.H. and Zhou, Z.J. (2012) Numerical Study on Effects of Inlet Structure of Radiant Syngas Cooler on Flow Field and Particle Concentration. Proceedings of the CSEE. 32, 15-21.

[3] Bohren, C.F. and Huffman, D.H. (1998) Absorption and Scattering of Light by Small Particles. Wiley-Interscience Publication. https://doi.org/10.1002/9783527618156

[4] Goodwin, D.G. (1986) Infrared Optical Constants of Coal Slags. Stanford University, Stanford.

[5] Goodwin, D.G. and Mitchner, M. (1989) Fly Ash and Effects on Radiative Heat Transfer in Coal-Fired System. Heat Mass Transfer, 32, 627-638. https://doi.org/10.1016/0017-9310(89)90211-1

[6] Self, S.A. (1992) Optical Properties of Fly Ash. Stanford University, Stanford.

[7] Liu, L.H., Yu, Q.Z., Ruan, L.M. and Tan, H.P. (1996) Radiation Properties of Pulverized Coal and Ash Particles. Journal of Combustion Science and Technology, 2, 127-133. https://doi.org/10.1080/00102209608951975

[8] Yu, Q.Z., Tan, H.P. and Ruan, L.M. (1993) The Optical Constant and Extinction Coefficient of Small Ash Particles. Journal of Engineering Thermophysics, 14, 449-452.

[9] Zheng, C.G. and Liu, C.H. (1996) Optical Properties and Radiative Heat Transfer of Dispersed Particles. HUST Press, Hubei.

[10] Yin, J.Y. and Liu, L.H. (2009) Effective Optical Constant and Radiative Property of Coal Slag. Journal of Engineering Thermophysics, 30, 115-117.

[11] Mengüc, M.P. (1994) Determination of Radiative Properties of Pulverized Coal from Experiments. Fuel, 73, 613-625. https://doi.org/10.1016/0016-2361(94)90048-5

[12] Gupta, R.P. and Wall, T.F. (1985) The Optical Properties of Fly Ash in Coal Fired Furnaces. Combustion \& Flame, 61, 145-151. https://doi.org/10.1016/0010-2180(85)90160-9

[13] Duan, M.H. and Fu, T.R. (2016) Uncertainty Analysis of Spectral Emissivity Measurement Method. Journal of Engineering Thermophysics, 37, 2684-2689.

[14] Tian, J.B. (2017) Measurement Method of Thermal Radiation Properties of Thermal Protection Materials. Ph.D. Thesis, Tsinghua University, Beijing.

[15] Fu, T.R., Tang, J. and Chen, K. (2016) Scattering and Absorption Coefficients of Silica-Doped Alumina Aerogels. Applied optics, 55, 705-711. https://doi.org/10.1364/AO.55.000705

[16] Tan, H.P., Xia, X.L., Liu, L.H. and Ruan, L.M. (2006) Numerical Calculation of 
Infrared Radiation and Transmission-Computational Thermal Radiation. Harbin Institute of Technology Press, Harbin.

[17] Liu, X.D. (2009) Measurement of the Complex Refractive Index of Particles Based on Mie Theory and Transmission Method. Infrared and Laser Engineering, 38, 820-824.

[18] Wang, L. and Eldridge, I.J. (2013) Comparison of Different Models for the Determination of the Absorption and Scattering Coefficients of Thermal Barrier Coatings. Acta Materialia, 64, 402-410. https://doi.org/10.1016/j.actamat.2013.10.053

[19] Eldridge, I.J. and Spuckler, C.M. (2015) Determination of Scattering and Absorption Coefficients for Plasma-Sprayed Yttria-Stabilized Zirconia Thermal Barrier Coatings. Journal of the American Ceramic Society, 91, 1603-1611. https://doi.org/10.1111/j.1551-2916.2008.02349.x

[20] Rozé, G. (2001) Average Crossing Parameter and Forward Scattering Ratio Values in Four-Flux Model for Multiple Scattering Media. Optics Communications, 194, 251-263. https://doi.org/10.1016/S0030-4018(01)01284-6

[21] Ruan, L.M., Yu, Q.Z., Liu, L.H. and Tan, H.P. (1995) Study on the Radiative Parameters of Coal and Ash. Journal of Engineering for Thermal Energy and Power, 10, 296-301. 